\title{
Magnetic Polaritons, Magnetostatic Waves and Effective-Medium Approximation for Antiferromagnetic Superlattice with Impurity in Parallel Magnetic Field
}

\author{
R.T. Tagiyeva (Askerbeyli) • B. Tanatar
}

Received: 8 July 2011 / Accepted: 11 July 2011 / Published online: 25 August 2011

(C) Springer Science+Business Media, LLC 2011

\begin{abstract}
We derive the general effective-medium expression for the surface-guided magnetic polaritons and magnetostatic waves, which propagate in the antiferromagnetic superlattice with antiferromagnetic impurity film, and investigate the influence of the external magnetic field on the energy of localized magnetic polaritons. Similarly as in the free-standing antiferromagnetic film, the spectrum of magnetic polaritons in the presence of an external magnetic field is reciprocal in the sense that the frequency is independent of the direction of propagation. In the system under consideration one finds both the surface polaritons which are strongly localized in the antiferromagnetic film which acts as a waveguide, and waves which are weakly localized within film. The first waves are the pure surface modes or guided modes where excitations have a standing-wavelike character. This important feature of the localized magnetic polaritons enables us to use these antiferromagnetic systems in the technologies for devices (for example, in resonators) that work at wavelengths in the infrared region. Second waves have the very small value of the decay parameter and appear in the regions where the surface mode penetrates into the bulk band, i.e. the magnetic polaritons are weakly localized in the impurity film region. Now we obtain the mixed type mode having both bulk and surface characteristics. Also, the general way in which the dispersion curves vary with the volume fraction of the superlattice components and with impurity film is illustrated in this study.
\end{abstract}

Keywords Magnetic polariton · Magnetostatic wave · Antiferromagnetic superlattice · Impurity · Dispersion relation

R.T. Tagiyeva (Askerbeyli) (凶) · B. Tanatar

Physics Department, Bilkent University, 06500, Ankara, Turkey

e-mail: Iman.Askerzade@ science.ankara.edu.tr

\section{Introduction}

The discovery and preparation of novel magnetic materials with unusual geometries and properties are the basic research stages in modern magnetism. The properties of the collective excitations such as polaritons (coupled-mode excitations originating from dipole-active elementary excitations such as phonons, plasmons, magnons, interacting with photons) which propagate in various magnetic (ferromagnetic, antiferromagnetic) superstructures as superlattices, have been the subject of increasing interest in recent years (see [1-8]). Also, excitations in magnetic films have been investigated by number of authors (see [9-12]).

During the past twenty years research efforts have been devoted to the investigation of different antiferromagneticbased superstructures and properties of the bulk and surface excitations propagating in such systems. Two approximations were applied for theoretical investigations of such systems: transfer matrix method [13] and effective-medium approximation (see [14-17]).

As is known, in contrast to ferromagnets, antiferromagnets can have long-wavelength spin excitations in the infrared frequency regime and thus superlattices constructed from antiferromagnetic materials are of interest to communications and signal processing technologies for devices that work at wavelengths in the infrared region (see [18, 19]).

Bulk and surface polaritons on antiferromagnetics were considered theoretically in $[20,21]$, and have been verified experimentally for $\mathrm{FeF}_{2}$ in $[18,19]$. The collective excitations such as polaritons, magnetostatic waves which propagate in the antiferromagnetic superstructures as superlattices, and thin films have attracted considerable attention during last decades (see [15, 22-24]).

In the earlier work [25] we derived the general dispersion relation for magnetic polaritons propagating in the mag- 


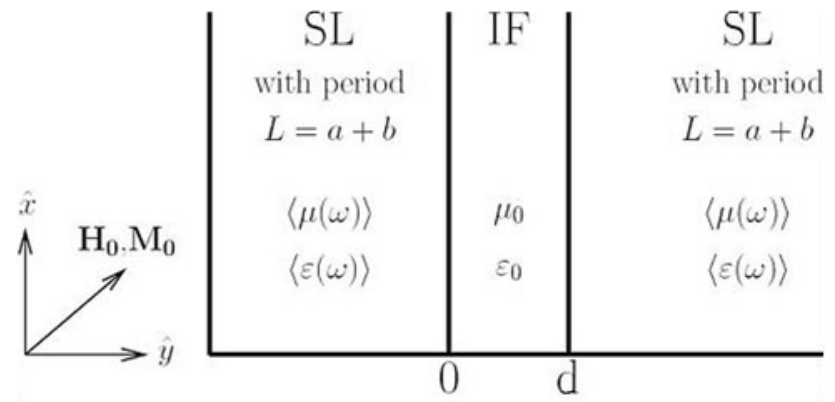

Fig. 1 The geometry considered in this paper. Here IF denotes impurity film and SL denotes antiferromagnetic superlattice

netic superlattice with magnetic impurity (defect) layer using transfer-matrix method and then applied the obtained dispersion relation to ferromagnetic superlattice. The aim of this paper is to extend our previous work on subject considering the propagation of magnetic polaritons which appear at the antiferromagnetic impurity film in the antiferromagnetic superlattice composed of alternating antiferromagnetic or antiferromagnetic and non-magnetic layers. This problem is considered within the framework of a macroscopic theory in the Voigt configuration in the presence of an external magnetic field using effective-medium approximation. Such a description is valid for antiferromagnetic superlattice because of the antiferromagnetic resonance frequencies are in the far infrared region and superlattice behaves like an anisotropic bulk medium. The presence of the impurity film leads to appearance of the localized modes (see [25-29]) and the impurity film can play the role of a waveguide. This important feature of the localized magnetic polaritons enables us to use these antiferromagnetic systems in magneto-optic device technology that work at wavelengths in the infrared region.

\section{Theory}

We consider the antiferromagnetic superlattice with period $L=a+b$ with thin antiferromagnetic impurity film of thickness $d$. All the layers are magnetized parallel to the film interface and parallel to the external magnetic field $\vec{H}_{0}$ and the $z$-axis. We restrict considerations to the Voigt geometry, i.e. to the propagation perpendicular to the spontaneous magnetization axis in the $x$-axis direction. The geometry of the system is shown in Fig. 1.

The effective-medium theory, in which both the dielectric tensor and the permeability tensor components are given as spatial averages of the dielectric and permeability constants of the constituent magnetic layers of the magnetic superlattices, can be applied in the regions of high-frequency dispersion, where wavelength is much greater than the superlattice period $(L=a+b)($ see [14-16]). Such a description is valid for antiferromagnetic superlattice because of the antiferromagnetic resonance frequencies are in the far infrared region and superlattice behaves like an anisotropic bulk medium.

Thus, the effective medium is described by the effectivemedium permeability tensor with the following components [14]:

$\mu_{x x}=\frac{\left((a+b)^{2} \mu_{x x}^{(1)} \mu_{x x}^{(2)}+a b\left[\left(\mu_{x x}^{(1)}-\mu_{x x}^{(2)}\right)^{2}+\left(\mu_{x y}^{(1)}-\mu_{x y}^{(2)}\right)^{2}\right]\right.}{(a+b)\left(a \mu_{x x}^{(2)}+b \mu_{x x}^{(1)}\right)}$,

$\mu_{x y}=\frac{a \mu_{x y}^{(1)} \mu_{x x}^{(2)}+b \mu_{x y}^{(2)} \mu_{x x}^{(1)}}{a \mu_{x x}^{(2)}+b \mu_{x x}^{(1)}}$

$\mu_{y y}=\frac{(a+b) \mu_{x x}^{(1)} \mu_{x x}^{(2)}}{a \mu_{x x}^{(2)}+b \mu_{x x}^{(1)}}$.

For the TE mode, the relevant component of the dielectric tensor in the effective-medium description is $\varepsilon_{\mathrm{sl}}$ :

$\varepsilon_{\mathrm{sl}}=\frac{a \varepsilon_{1}+b \varepsilon_{2}}{a+b}$

where $\varepsilon_{i}(i=1,2)$ is the dielectric constant of the $i$ th component of the superlattice. Here $\mu_{1}^{(i)}$ and $\mu_{2}^{(i)}$ are the nonvanishing components of the frequency-dependent magnetic permeability tensor:

$\overleftrightarrow{\mu}^{(i)}(\omega)=\left[\begin{array}{ccc}\mu_{1}^{(i)} & i \mu_{2}^{(i)} & 0 \\ -i \mu_{2}^{(i)} & \mu_{1}^{(i)} & 0 \\ 0 & 0 & 1\end{array}\right]$,

and for an antiferromagnet the elements of $\overleftrightarrow{\mu}^{(i)}(\omega)$ are

$\mu_{1}(\omega)=1+\frac{\Omega_{a} \Omega_{m}}{\Omega_{1}^{2}-\omega_{+}^{2}}+\frac{\Omega_{a} \Omega_{m}}{\Omega_{1}^{2}-\omega_{-}^{2}}$,

$\mu_{2}(\omega)=\frac{\Omega_{a} \Omega_{m}}{\Omega_{1}^{2}-\omega_{+}^{2}}-\frac{\Omega_{a} \Omega_{m}}{\Omega_{1}^{2}-\omega_{-}^{2}}$,

where $\Omega_{m}=\gamma M_{0}\left(M_{0}\right.$ is the sublattice magnetization and $\gamma=g \mu_{0} \gamma_{0}$; where $g$ and $\mu_{0}$ denote the Lande factor and magnetic permeability of the vacuum, respectively, and $\gamma_{0}=\frac{e}{2 m}$ with $e$ and $m$ being the electron charge and electron mass), $\Omega_{0}=\gamma H_{0}$ and $\omega_{ \pm}=\omega \pm \Omega_{0}$. The antiferromagnetic resonance frequency in zero applied field $\Omega_{1}$ is given by the anisotropy $H_{\mathrm{an}}$ and the exchange $H_{\mathrm{ex}}$ fields as

$\Omega_{1}=\gamma\left[H_{\mathrm{an}}\left(2 H_{\mathrm{ex}}+H_{\mathrm{an}}\right)\right]^{\frac{1}{2}}$.

The dynamic magnetic field $\vec{H}(\vec{r}, t)$ satisfies the following equation which follows from Maxwell's equations after eliminating $\vec{E}(\vec{r}, t)$ in curl equations:

$\nabla^{2} \vec{H}-\vec{\nabla}(\vec{\nabla} \vec{H})-\frac{\varepsilon}{c^{2}} \frac{\partial^{2}}{\partial t^{2}}(\vec{H}+4 \pi \vec{m})=0$. 
To derive the dispersion relation for the magnetic polaritons in the effective-medium limit, we write the field in the following form:

$\vec{H}_{\mathrm{sl}}=\left(\left\langle H_{x}^{\mathrm{sl}}\right\rangle,\left\langle H_{y}^{\mathrm{sl}}\right\rangle\right) e^{\beta y} e^{i(k x-\omega t)}, \quad y<0$,

$\vec{H}_{\mathrm{sl}}=\left(\left\langle H_{x}^{\mathrm{sl}}\right\rangle,\left\langle H_{y}^{\mathrm{sl}}\right\rangle\right) e^{-\beta y} e^{i(k x-\omega t)}, \quad y>d$

for SL and

$\vec{H}^{(0)}=\left[\left(H_{1 x}^{(0)}, H_{1 y}^{(0)}\right) e^{\alpha_{0} y}+\left(H_{2 x}^{(0)}, H_{2 y}^{(0)}\right) e^{-\alpha_{0} y}\right] e^{i(k x-\omega t)}$

for the impurity film occupying the region $0<y<d$.

Here $\beta^{2}=-k_{y}^{2}$ and

$\alpha_{0}^{2}=k^{2}-\varepsilon_{0} \mu_{v}^{(0)} \omega^{2} / c^{2}$.

Furthermore, $\left\langle H_{x, y}^{\mathrm{sl}}\right\rangle,\left\langle B_{x, y}^{\mathrm{sl}}\right\rangle$ are the average values of $H_{x, y}^{\mathrm{sl}}$, $B_{x, y}^{\mathrm{sl}}$ in the superlattice.

The determination of the surface-guided magnetic polariton dispersion relation requires the imposition of the electromagnetic boundary conditions at the surfaces of the impurity film, $y=0$ and $y=d$, namely, the continuity of the tangential component of the magnetic field $\vec{H}$ and normal component of $\vec{B}$. After a bit of algebra, we obtain:

$$
\begin{aligned}
A\left[\mu_{1}^{(0)} \alpha_{0} \cosh \left(\alpha_{0} d\right) B_{1}+\sinh \left(\alpha_{0} d\right) B_{2}\right]=0, & \\
B_{1}= & 2 \beta \mu_{y y}\left(\varepsilon_{\mathrm{sl}} \frac{\omega^{2}}{c^{2}} \mu_{y y}-k^{2}\right), \\
B_{2}= & \left(k \mu_{2}+\beta \mu_{y y}\right)\left\{k \mu_{2}^{(0)}\left(\varepsilon_{\mathrm{sl}} \frac{\omega^{2}}{c^{2}} \mu_{y y}-k^{2}\right)\right. \\
& \left.-\left(k \mu_{2}-\beta \mu_{y y}\right)\left(\varepsilon_{0} \frac{\omega^{2}}{c^{2}} \mu_{1}^{(0)}-k^{2}\right)\right\} \\
& +\left(\varepsilon_{\mathrm{sl}} \frac{\omega^{2}}{c^{2}} \mu_{y y}-k^{2}\right)\left\{k \mu_{2}^{(0)}\left(k \mu_{2}-\beta \mu_{y y}\right)\right. \\
& \left.-\mu_{\nu}^{(0)} \mu_{1}^{(0)}\left(\varepsilon_{\mathrm{sl}} \frac{\omega^{2}}{c^{2}} \mu_{y y}-k^{2}\right)\right\}, \\
A^{-1}= & \left(\varepsilon_{\mathrm{sl}} \frac{\omega^{2}}{c^{2}} \mu_{y y}-k^{2}\right)^{2}\left(\varepsilon_{0} \frac{\omega^{2}}{c^{2}} \mu_{1}^{(0)}-k^{2}\right) .
\end{aligned}
$$

Here $\mu_{x y}=i \mu_{2}$ and the coefficient $\beta$ is the decay parameter of the magnetic polaritons in the superlattice. The expression for the parameter $\beta$ can be obtained from the dispersion relation for bulk magnetic polaritons in the effective medium [14]:

$\beta^{2}=k^{2}\left[f_{a}^{2}+f_{b}^{2}+f_{a} f_{b} \frac{\mu_{v}^{(1)} \mu_{1}^{(1)}+\mu_{v}^{(2)} \mu_{1}^{(2)}+2 \mu_{2}^{(1)} \mu_{2}^{(2)}}{\mu_{1}^{(1)} \mu_{1}^{(2)}}\right]$

$$
-\frac{\omega^{2}}{c^{2}}\left(f_{a} \varepsilon_{1}+f_{b} \varepsilon_{2}\right)\left(f_{a} \mu_{v}^{(1)}+f_{b} \mu_{v}^{(2)}\right)
$$

where $f_{a}=a /(a+b), f_{b}=b /(a+b)$.

Equations (14)-(17) together with (18) determine the frequencies of the localized magnetic polaritons propagating in the system consisting of antiferromagnetic superlattice with antiferromagnetic film in the effective-medium description. This equation is the general dispersion relation for surfacelocalized magnetic polaritons propagating parallel to the impurity film and perpendicular to the magnetic moments and to the applied external magnetic field (Voigt geometry) and can be applied to both ferromagnetic and antiferromagnetic systems.

In thin impurity films, one finds both surface polaritons in which the excitation is localized near the surface, and guided modes, with an oscillatory profile for the field inside the film. Thus the parameter $\alpha_{0}$ in (13) can now be either purely real (for surface modes) or purely imaginary (for guided modes). In order to have a bounded excitation we also require the wave vector $\beta$ be real and positive.

In the magnetostatic limit $k^{2} \gg \omega^{2} / c^{2}$, the decay parameters reduce to $\alpha_{0}=k$ and

$$
\begin{aligned}
\beta= & k\left[f_{a}^{2}+f_{b}^{2}\right. \\
& \left.+f_{a} f_{b} \frac{\mu_{v}^{(1)} \mu_{1}^{(1)}+\mu_{v}^{(2)} \mu_{1}^{(2)}+2 \mu_{2}^{(1)} \mu_{2}^{(2)}}{\mu_{1}^{(1)} \mu_{1}^{(2)}}\right]^{1 / 2} \\
= & k\left(\frac{\mu_{x x}}{\mu_{y y}}\right)^{1 / 2} .
\end{aligned}
$$

Equation (14) then reduces to

$$
\begin{aligned}
& 2\left(\mu_{x x} \mu_{y y}\right)^{1 / 2} \mu_{1}^{(0)}-\tanh (k d)\left(\mu_{2}+\left(\mu_{x x} \mu_{y y}\right)^{1 / 2}\right) \\
& \quad \times\left\{\mu_{2}-\mu_{2}^{(0)}-\left(\mu_{x x} \mu_{y y}\right)^{1 / 2}\right\} \\
& \quad \times \mu_{2}^{(0)}\left(\mu_{2}-\left(\mu_{x x} \mu_{y y}\right)^{1 / 2}\right)-\mu_{\nu}^{(0)} \mu_{1}^{(0)}=0 .
\end{aligned}
$$

One can analyze (20) in the following special case, when $k d \ll 1$. The hyperbolic tangent in (20) tends to $k d$, hence we have the explicit solution for $k$ :

$k=\frac{-2 \mu_{1}^{(0)}}{d\left[\left(\mu_{1}^{(0)}\right)^{2}-\left(\mu_{2}^{(0)}\right)^{2}+1\right]}$.

This result holds for very thin films.

\section{Results and Discussion}

The obtained analytical results we apply to the antiferromagnetic superlattice $\mathrm{SL}\left(\mathrm{MnF}_{2} / \mathrm{ZnF}_{2}\right)$ with antiferromagnetic impurity film $\mathrm{FeF}_{2}$ with thickness $d=0.0005 \mathrm{~cm}$. 


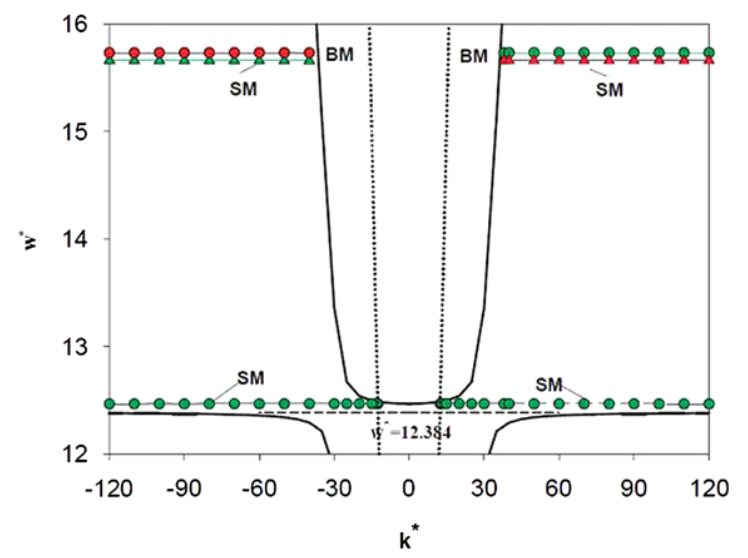

Fig. 2 Dispersion relation for bulk and surface (guided) polaritons in $\mathrm{MnF}_{2}$ antiferromagnet in $H=0, f_{a}=1, f_{b}=0$

In the following figures we present the magnetic polariton spectra. The different symbols (open and solid squares, triangles, dots) denote the surface (SM) and guided (GM) modes. Here we use the following parameters for $\mathrm{MnF}_{2}$ : $\mu_{0} H_{\mathrm{ex}}=55 \mathrm{~T}, \mu_{0} H_{\mathrm{an}}=0.787 \mathrm{~T}, \gamma=4.5, \mu_{0} M_{0}=0.754 \mathrm{~T}$, $\varepsilon=5.5$ and $\mu_{0} H_{\mathrm{ex}}=54 \mathrm{~T}, \mu_{0} H_{\mathrm{an}}=20 \mathrm{~T}, \mu_{0} M_{0}=0.624 \mathrm{~T}$, $\gamma=1.05, \varepsilon=5.5$ for $\mathrm{FeF}_{2}$ [2]. For the non-magnetic material we take $\varepsilon=8$. For numerical calculations we have introduced the following dimensionless parameters: $\Omega_{0}^{*}=$ $\Omega_{0} / \Omega_{m}^{\left(\mathrm{MnF}_{2}\right)}, \omega^{*}=\omega / \Omega_{m}^{\left(\mathrm{MnF}_{2}\right)}, k^{*}=c k / \Omega_{m}^{\left(\mathrm{MnF}_{2}\right)}, d^{*}=$ $\Omega_{m}^{\left(\mathrm{MnF}_{2}\right)} d / c$, and the magnetic polariton spectra are presented through a plot of the reduced frequency $\omega^{*}$ against the wavevector $k^{*}$.

Firstly, we consider the case $f_{a}=1$ and $f_{b}=0$, corresponding to the bulk antiferromagnet with antiferromagnetic impurity film in zero applied field and $\mu_{0} H_{0}=0.3 \mathrm{~T}$ (Figs. 2-4). In the absence of the external magnetic field we observe three localized surface (and guided) mode branches of magnetic polaritons for each direction of $k^{*}$. Two highfrequency surface modes exist between the bulk bands of $\mathrm{FeF}_{2}$ and start at $\omega_{1}^{*\left(\mathrm{FeF}_{2}\right)}=15.6576\left(\frac{\omega}{g \gamma_{0}}=11.8058 \mathrm{~T}\right)$ at the finite value of the wavevector (where $\omega_{1}^{*\left(\mathrm{FeF}_{2}\right)}$ is the antiferromagnetic resonance frequency in $H_{0}=0$ and is determined by (8)). Low-frequency SM branches (see Fig. 2) split at the bottom of the high bulk band at the frequency $\omega^{*}=12.467\left(\frac{\omega}{g \gamma_{0}}=9.4001 T\right)$ and $k^{*}=12.5$ with imaginary $\alpha_{0}$ as guided wave (GM) but with increasing $\left|k^{*}\right|$ transforms to the surface mode and tends to the frequency $\omega^{*}=12.4612\left(\frac{\omega}{g \gamma_{0}}=9.3957 \mathrm{~T}\right)$. The frequency of the surface (guided) mode does not depend on the sign of the wavevector, i.e., the surface modes are reciprocal.

Figures 3-4 show the dispersion curves of the surfaceguided modes for the same structure with $\mu_{0} H_{0}=0.3 \mathrm{~T}$. In contrast to the above, there are now three bulk bands and in the presence of an external magnetic field the new localized modes appear making the spectra more complex.
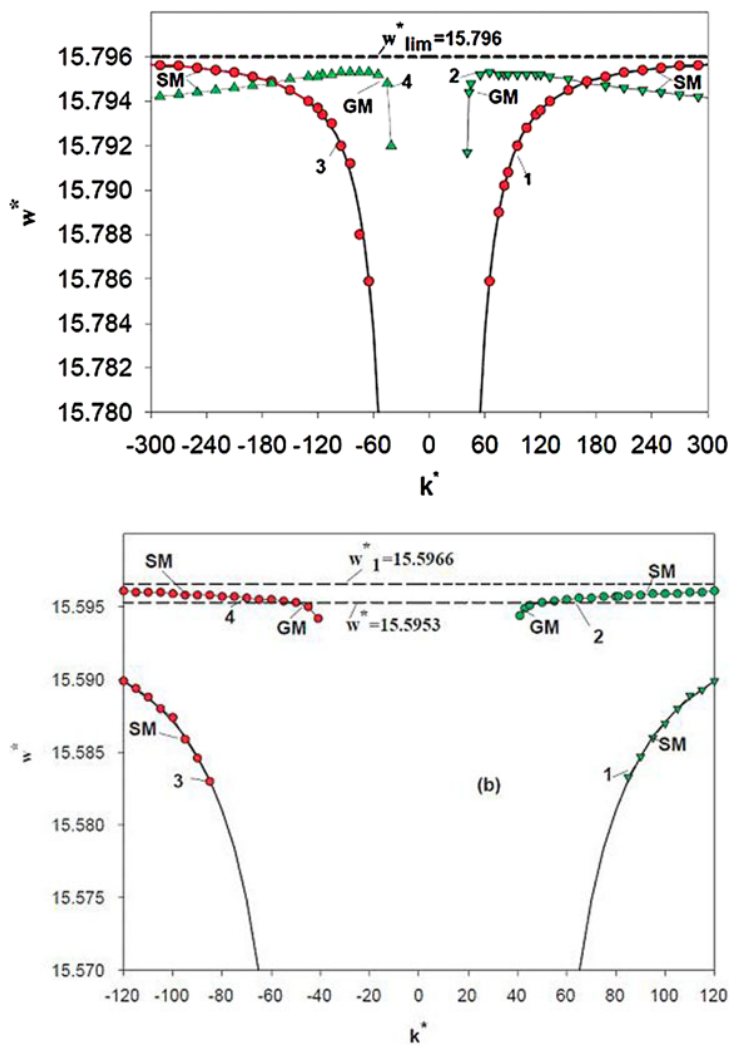

Fig. 3 Dispersion relation for bulk and surface (guided) polaritons in $\mathrm{FeF}_{2}$ antiferromagnet in $H=0.3, f_{a}=1, f_{b}=0, f_{a}=0.8, f_{b}=0.2$ and $f_{a}=0.5, f_{b}=0.5$ : (a) High-frequency bulk and surface (guided) modes of magnetic polaritons in antiferromagnet $\mathrm{FeF}_{2}$. (b) The dispersion relation for low-frequency bulk and surface (guided) modes of polaritons in $\mathrm{FeF}_{2}$ antiferromagnet

Figures 3-4 for $f_{a}=1, f_{b}=0$ in comparison with Fig. 2, show the way in which the dispersion curves depend on the magnetic field. It is seen that in the presence of an external magnetic field there is the appearance of the new bulk bands at $\omega^{*}=15.795\left(\frac{\omega}{g \gamma_{0}}=11.9094 \mathrm{~T}\right)$ and $\omega^{*}=15.595$ $\left(\frac{\omega}{g \gamma_{0}}=11.7586 \mathrm{~T}\right)$ and a new surface mode close to those. In the regions where the surface mode penetrates into the bulk band we see mixed-type modes having both bulk and surface characteristics. These modes are denoted as 1 and 3 in Fig. 3(a, b)). Also there are modes (denoted as 2 and 4 in Fig. 3(a, b)) which start as a guided modes (with complex $\alpha_{0}$ ) and exist in the restricted wavevector and frequency range $\omega^{*}=15.7917-15.7948\left(\frac{\omega}{g \gamma_{0}}=11.9069-11.9093 \mathrm{~T}\right)$ $\left(k^{*}=41-170\right)$ for high-frequency branches (see Fig. 3(a)) and $\omega^{*}=15.5944-15.5953\left(\frac{\omega}{g \gamma_{0}}=11.75818-11.7588 \mathrm{~T}\right)$ $\left(k^{*}=41-50\right)$ for low-frequency branches (see Fig. 3(b)). With increasing $k^{*}\left(k^{*} \rightarrow+\infty\right)$ these curves transform to the surface modes with real $\alpha_{0}$ at $\omega^{*}=15.7948\left(\frac{\omega}{g \gamma_{0}}=\right.$ $11.90928 \mathrm{~T})$ and $\omega^{*}=15.5953\left(\frac{\omega}{g \gamma_{0}}=11.7588 \mathrm{~T}\right)$, respectively. The frequencies $\omega_{\lim }^{*}=15.796\left(\frac{\omega}{g \gamma_{0}}=11.91018 \mathrm{~T}\right)$ and $\omega_{1}^{*}=15.5966\left(\frac{\omega}{g \gamma_{0}}=11.75983 \mathrm{~T}\right)$ in parts (a) and (b) of Fig. 3 are the limiting frequencies for high and low bulk 

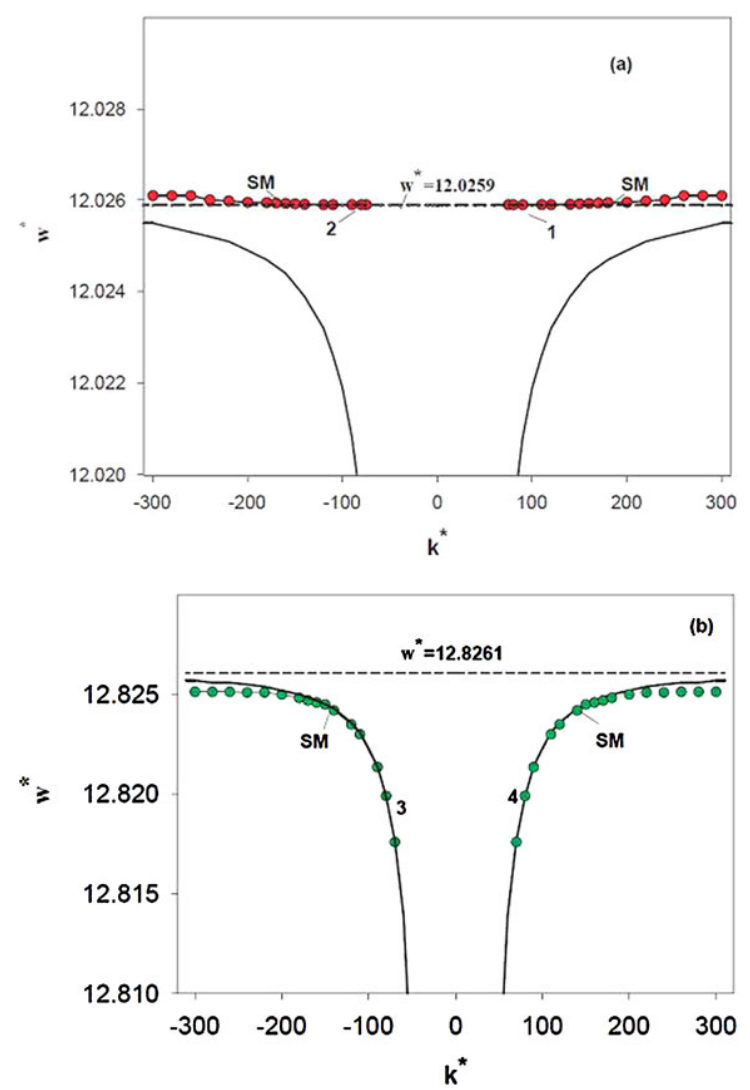

Fig. 4 Dispersion relation for bulk and surface (guided) polaritons in bulk $\mathrm{MnF}_{2}$ antiferromagnet $H=0.3, f_{a}=1, f_{b}=0$ : (a) The dispersion curve for low-frequency bulk and surface (guided) modes of polaritons. (b) High-frequency bulk and surface (guided) modes of magnetic polaritons

continuum, respectively. It is seen that in the presence of an external magnetic field the number of the localized branches increases: for example, in general we observe six surfaceguided modes of localized magnetic polaritons for both directions of the wavevector, respectively: four curves in the $\mathrm{FeF}_{2}$-resonance region (see Fig. 3(a, b)) and two surface modes in the $\mathrm{MnF}_{2}$-resonance region (see Fig. 4(a, b)). The curves denoted as 1 and 2 in Fig. 4(a) appear at $k^{*}=75$ and at the frequency $\omega^{*}=12.0259$ and simultaneously with a small group vector and with very much value of $\beta\left((\operatorname{Re} \beta)^{-1}\right.$ is the penetration depth in the superlattice). Second surface wave splits from the bulk continuum at $k^{*}=70$ and $\omega^{*}=12.8176\left(\frac{\omega}{g \gamma_{0}}=9.66447 \mathrm{~T}\right)$ (see Fig. 4(b)) and lies under the limiting frequency $\omega^{*}=12.8261\left(\frac{\omega}{g \gamma_{0}}=9.67088 \mathrm{~T}\right)$. These waves are the pure surface modes strongly localized on the impurity film.

Now we consider the case of superlattice with $f_{a}=0.8$ and $f_{b}=0.2$ in the magnetic field $\mu_{0} H_{0}=0$ and $0.3 \mathrm{~T}$. In the absence of an external magnetic field the curves are similar to these for the case $f_{a}=1, f_{b}=0, \mu_{0} H_{0}=0$ (see Fig. 2), except that the lower-frequency wave branch originates from the bulk branch at $\omega^{*}=12.45123\left(\frac{\omega}{g \gamma_{0}}=\right.$
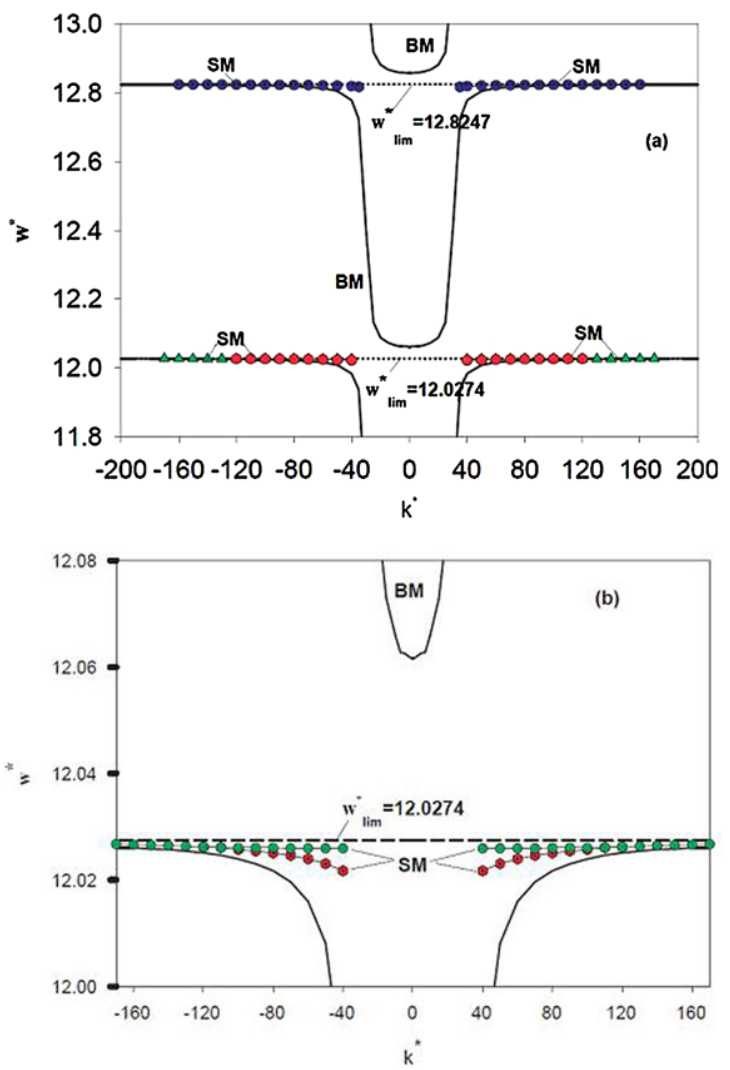

Fig. 5 (a) Bulk and surface modes of magnetic polaritons in antiferromagnetic superlattice $\mathrm{SL}\left(\mathrm{MnF}_{2} / \mathrm{ZnF}_{2}\right)$ with applied field $H=0.3 \mathrm{~T}$ for $f_{a}=0.8, f_{b}=0.2$. (b) Same as in (a), in another scale

$9.3882 \mathrm{~T}$ ) as guided mode and with increasing $k$ the frequency decreases, so this branch occupies a narrow range of $\omega^{*}=12.4008-12.45123\left(\frac{\omega}{g \gamma_{0}}=9.3502-9.3882 \mathrm{~T}\right)$ (here the limiting frequency is $\omega^{*}=12.4008\left(\frac{\omega}{g \gamma_{0}}=9.3502\right)$. For an external magnetic field equal to $0.3 \mathrm{~T}$, we have three localized mode branches in the $\mathrm{MnF}_{2}$-resonance region which lie in the frequency range between the limiting frequency and the bulk continuum (see Fig. 5(a)). As can be seen from Fig. 5(b), in the low-frequency region the branch splits into two branches which exist under the limiting frequency $\omega^{*}=12.0274\left(\frac{\omega}{g \gamma_{0}}=9.0687 \mathrm{~T}\right)$. It is essential that one of these branches is short and exists for $k^{*}=40-160$ and $\omega^{*}=12.0217-12.02617\left(\frac{\omega}{g \gamma_{0}}=9.06436-9.0677 \mathrm{~T}\right)$. The second curve starts at $\omega^{*}=12.02589\left(\frac{\omega}{g \gamma_{0}}=9.0675 \mathrm{~T}\right)$ and with increasing $k^{*}$ penetrates into the bulk band as well as high-frequency surface mode which appears at frequency $\omega^{*}=12.81684\left(\frac{\omega}{g \gamma_{0}}=9.6639 \mathrm{~T}\right)$ with very much value of $\beta$.

For superlattice with $f_{a}=f_{b}=0.5$ and $\mu_{0} H_{0}=0$, the general picture does not change: there are two highfrequency surface modes in the $\mathrm{FeF}_{2}$-resonance region which coincide with previous cases in zero field (see Fig. 2), but in the $\mathrm{MnF}_{2}$-resonance region now the low-frequency 
branch starts as guided mode for small value of wavevector at the frequency $\omega^{*}=12.4261\left(\frac{\omega}{g \gamma_{0}}=9.3693 \mathrm{~T}\right)$ and then transforms to the surface mode. Similarly as in the previous cases, the spectrum of the surface (guided) modes in zero field is reciprocal. In the presence of external magnetic field $\mu_{0} H_{0}=0.3 T$ the frequency curves in the $\mathrm{FeF}_{2}$ resonance range do not change with the varying of volume fraction parameters $f_{a}$ and $f_{b}$ (see Fig. 3(a, b)), but in contrast to the above, the surface modes in the $\mathrm{MnF}_{2}$-resonance region denoted as 1 and 2 in Fig. 6 now exist for the restricted values the wavevector $\left(k^{*}=75-100\right)$ at the frequency $\omega^{*}=12.0259\left(\frac{\omega}{g \gamma_{0}}=9.06752 \mathrm{~T}\right)$ and the second branch is close to bulk continuum and tends to limiting frequency $\omega_{\text {lim }}^{*}=12.0282\left(\frac{\omega}{g \gamma_{0}}=9.06926 \mathrm{~T}\right)$.

Now we want to consider the dependence of the dispersion curves on the impurity film material. The following physical parameters for antiferromagnetic impurity film are used: $\mu_{0} H_{\mathrm{ex}}=54 \mathrm{~T}, \mu_{0} H_{\mathrm{an}}=1.005 * 20 \mathrm{~T}, \mu_{0} M_{0}=$ $2 * 0.624 \mathrm{~T}, \gamma=1.05, \varepsilon_{0}=5.5$ and $H_{0}=0.3 \mathrm{~T}$. The nu-

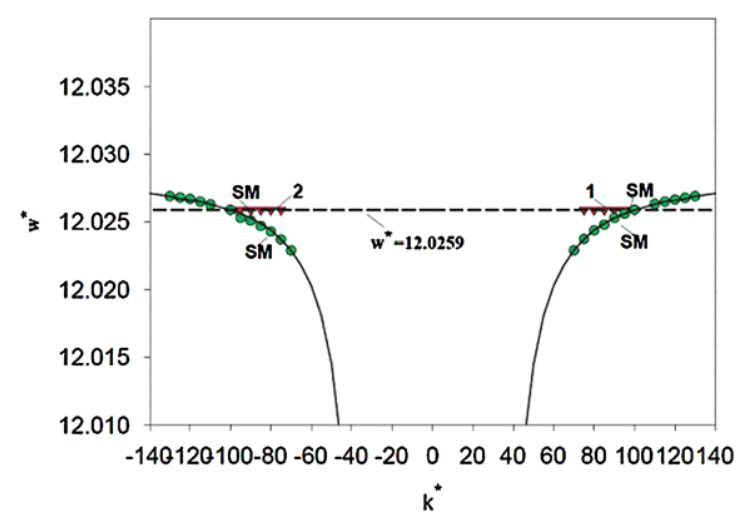

Fig. 6 Low-frequency bulk and surface (guided) modes of magnetic polaritons in antiferromagnetic superlattice $\mathrm{SL}\left(\mathrm{MnF}_{2} / \mathrm{ZnF}_{2}\right)$ with applied field $H=0.3$ T for $f_{a}=0.5, f_{b}=0.5$

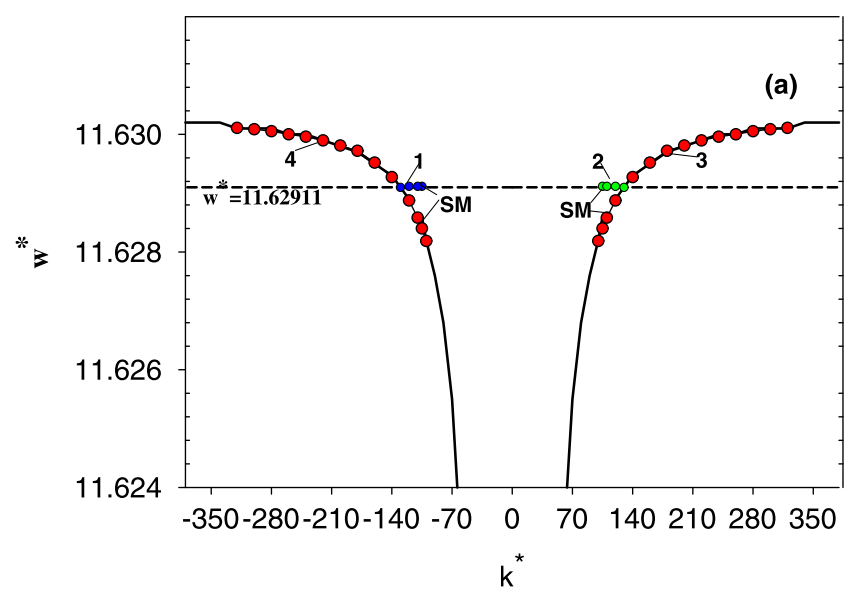

merical calculations show that the frequency region of the existence of the localized magnetic polaritons in the $\mathrm{MnF}_{2}$ resonance range does not change with varying the physical parameters of the impurity film (see Fig. 6), in contrast to the modes which now appear in the $\mathrm{FeF}_{2}$-resonance region at the frequencies $\omega^{*}=15.899\left(\frac{\omega}{g \gamma_{0}}=11.9878 \mathrm{~T}\right)$ and $\omega^{*}=15.6594\left(\frac{\omega}{g \gamma_{0}}=11.8072 \mathrm{~T}\right)$, respectively, although the general behavior is the same (see Fig. 3). So as the impurity film magnetization increases, the high frequency of localized modes increases also, but at the same time the lowfrequency modes do not change. It is essential that the general picture does not vary.)

Finally, we want illustrate the numerical results for the system under consideration in magnetic field $\mu_{0} H_{0}=0.6 \mathrm{~T}$. In this case the surface-guided waves in the $\mathrm{FeF}_{2}$-resonance range exist at frequency $\omega_{\lim }^{*}=15.8852\left(\frac{\omega}{g \gamma_{0}}=11.9774 \mathrm{~T}\right)$ for high-frequency and $\omega^{*}=15.5063\left(\frac{\omega}{g \gamma_{0}}=11.6917 \mathrm{~T}\right)$ for low-frequency curves. From the numerical calculations we can conclude that as the external field increases, the frequency of the surface-guided high-frequency waves increases. At the same time the frequency interval (the difference between the low $-\omega^{*}$ and high- $\omega^{*}$ surface wave branches) increases. Thus now the surface wave branches exist in the $\mathrm{MnF}_{2}$-resonance range in the restricted interval of $k^{*}=105-130$ at $\omega^{*}=11.629116-11.62912\left(\frac{\omega}{g \gamma_{0}}=\right.$ 8.7683-8.76835 T) (denoted as 1 and 2 in Fig. 7(a)) and the branches 3 and 4 start at $\omega^{*}=11.6282\left(\frac{\omega}{g \gamma_{0}}=8.76766 \mathrm{~T}\right)$. The high-frequency curves degenerate at value of $k^{*}=110$ at $\omega^{*}=13.2195\left(\frac{\omega}{g \gamma_{0}}=9.9675 \mathrm{~T}\right)($ see Fig. $7(\mathrm{~b}))$.

\section{Conclusion}

In summary, we have derived the general effective-medium expression for the surface-guided magnetic polaritons, which

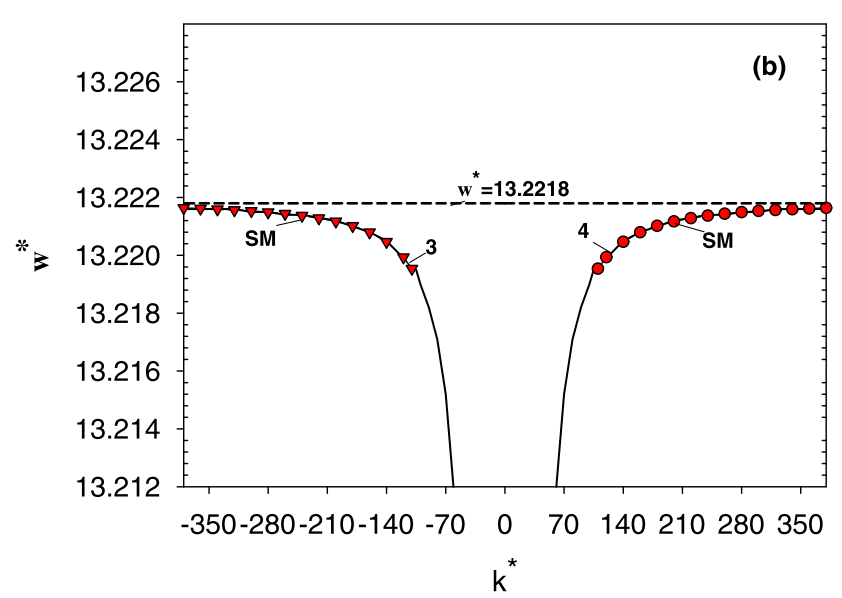

Fig. 7 (a) Low-frequency bulk and surface (guided) modes of magnetic polaritons in antiferromagnetic superlattice $\mathrm{SL}\left(\mathrm{MnF}_{2} / \mathrm{ZnF} 2\right)$ with applied field $H=0.6$ T for $f_{a}=0.5, f_{b}=0.5$. (b) Same as in (a), for high-frequency modes of magnetic polaritons 
propagate in the antiferromagnetic superlattice with antiferromagnetic impurity film and investigate the influence of the external magnetic field on the energy of localized magnetic polaritons. Similarly as in the free-standing antiferromagnetic film, the spectrum of magnetic polaritons in the presence of an external magnetic field is reciprocal in the sense that the frequency is independent of the direction of propagation.

In the system under consideration one finds both the surface polaritons which are strongly localized in the antiferromagnetic film which acts as a waveguide, and waves which are weakly localized within film. The first waves are the pure surface modes or guided modes where excitations have a standing-wave-like character. This important feature of the localized magnetic polaritons enables us to use these antiferromagnetic systems in the technologies for devices (for example, for resonators) that work at wavelengths in the infrared region. Second waves have the very small value of the parameter $\beta$ and appear in the regions where the surface mode penetrates into the bulk band, i.e., the magnetic polaritons are weakly localized in the impurity film region. Now we obtain the mixed type modes having both bulk and surface characteristics. Also the general way in which the dispersion curves vary with the volume fraction of the superlattice components and with impurity film is illustrated in this paper.

We hope that our theoretical predictions will motivate further experimental work.

Acknowledgements R.T. is supported by TUBITAK-BIDEP and she thanks the hospitality of the Department of Physics, Bilkent University. B.T. is supported by TUBITAK (108T743), TUBA, and EUFP7 project UNAM-REGPOT (203953).

\section{References}

1. Camley, R.E.: J. Magn. Magn. Mater. 200, 583-597 (1999)

2. Barnas, J.: J. Phys., Condens. Matter 2, 7173-7180 (1990)

3. Tagiyeva, R.T.: Superlattices Microstruct. 35, 9-17 (2004)
4. Schuller, I.K., Kim, S., Leighton, C.: J. Magn. Magn. Mater. 200, 571-582 (1999)

5. Barnas, J.: Solid State Commun. 61, 405-408 (1987)

6. Agranovich, V.M., Kravtsov, V.E.: Solid State Commun. 55, 8590 (1985)

7. Albuquerque, E.L., Fulco, P., Sarmento, E., Tilley, D.R.: Solid State Commun. 58, 41-44 (1986)

8. Camley, R.E., Cottam, M.G.: Phys. Rev. B 35, 189-196 (1987)

9. Damon, R.W., Eshbach, J.R.: J. Phys. Chem. Solids 19, 308-320 (1961)

10. Karsono, A.D., Tilley, D.R.: J. Phys. C, Solid State Phys. 11, 3487-3492 (1978)

11. Lima, N.P., Oliveira, F.A.: J. Phys. C, Solid State Phys. 19, 53815392 (1986)

12. Kaganov, M.I., Pustylnik, N.B., Shalayeva, T.I.: Phys. Usp.. 40(2), 181-224 (1997)

13. Elmzughi, F.G., Camley, R.E.: J. Phys., Condens. Matter 9, 10391048 (1997)

14. Raj, N., Tilley, D.: Rhys. Rev. B 36, $7003-7007$ (1987)

15. Almeida, N.S., Tilley, D.R.: Solid State Commun. 73, 23-27 (1990)

16. Elmzughi, F.G., Constantinou, N.C., Tilley, D.R.: Phys. Rev. B 51, 17 (1995), 11515-11520

17. Tagiyeva, R., Saglam, M., Boyacioglu, B.: J. Phys. Chem. Solids 68, 1296-1304 (2007)

18. Biehlig, W., Lederer, F., Trutschel, U.: J. Quantum Electron. 28(7), 1643-1646 (1992)

19. Brown, D.E., Dumelow, T., Parker, T.J., Abraha, K., Tilley, D.R.: Phys. Rev. B 49, 12266-12269 (1994)

20. Abraha, K., Brown, D.E., Dumelow, T., Parker, T.J., Tilley, D.R.: Phys. Rev. B 50, 6808-6816 (1994)

21. Jensen, M.R.F., Feiven, S.A., Parker, T.J., Camley, R.E.: Phys. Rev. B 55, 2745-2748 (1997)

22. Stamps, R., Camley, R.: Phys. Rev. B 40, 596-608 (1989)

23. Stamps, R.L., Camley, R.E.: Phys. Rev. B 40, 609-621 (1989)

24. Camley, R.E., Cottam, M.G., Tilley, D.R.: Solid State Commun. 81, 571-574 (1992)

25. Oliveros, M.C., Almeida, N.S., Tilley, D.R., Thomas, J., Camley, R.E.: J. Phys., Condens. Matter 4, 8497-8510 (1992)

26. Guimaraes, E., Albuquerque, E.: Solid State Commun. 122, 623$628(2002)$

27. Tagiyeva, R.T.: Phys. Scr. 70, 202-206 (2004)

28. Amir, A., Friberg, J., Friberg, A. T.: Opt. Lett. 30(10), 1213-1215 (2005)

29. Inoue, M., Fujikawa, R., Baryshev, A., Khanikaev, A., Lim, P.B., Uchida, H., Aktsipetrov, O., et al.: J. Phys. D, Appl. Phys. 39, R151-R161 (2006) 